\title{
LOS SESGOS COGNITIVOS EN LA TOMA DE DECISIONES
}

\author{
Nuria Cortada de Kohan \\ Profesora Honoraria de la Universidad de Buenos Aires, Argentina
}

\begin{abstract}
This paper reviews the "Heuristics and biases" program initiated by Tversky \& Kahneman in 1970, and thanks to which Daniel Kahneman shared the Economic Sciences Nobel Prize in 2002. These authors have proved that there are a lot of biases in individuals' intuitions about the potential occurrence of any event. The paper describes several biases and gives examples where this statement can be observed including the base rate fallacy and the conjunction fallacy to mention only but a few. In addition, this work underlines the complex problem of decision-making in uncertainty cases, reviews the prospect theory and the overconfidence bias this paper's author is presently working on.
\end{abstract}

Keywords: Uncertainty, cognitive biases, decision-making, overconfidence.

\section{RESUMEN}

En este trabajo se hace una presentación del Programa de "Heurísticos y sesgos" que iniciaron Tversky y Kahneman en el año 1970 y que en el 2002 le valió a Daniel Kahneman compartir el Premio Nobel de Economía. Estos autores han demostrado que en las intuiciones de las personas sobre la probabilidad de los hechos se producen muchos sesgos y, en este trabajo, se señalan algunos de ellos y se dan los ejemplos usados para ello, como la falacia de la ley de los pequeños números, el sesgo de conjunción y otros Se señala, además, el complejo problema de la toma de decisiones en los casos de incertidumbre, se presenta la teoría de las expectativas (prospect theory) y el sesgo de sobre confianza en el que actualmente trabaja la autora de este artículo.

Palabras Clave: incertidumbre, sesgos cognitivos, toma de decisiones, sobre confianza.

A lo largo de la vida todas las personas tomamos una gran cantidad de decisiones; algunas veces son fáciles y, por el contrario, otras son muy difíciles de tomar porque nos encontramos frente a una duda, conflicto o incertidumbre. A veces, la incertidumbre proviene de no saber qué va a pasar en el mundo, como cuando no estamos seguros de si va a llover o no y por tanto si decidir salir con paraguas o no y otras veces la incertidumbre está en la falta de conocimiento de uno mismo, "si concurro a un restaurante chino ¿Me gustará la comida?, si elijo tal carrera "¿'Tendré suficientes aptitudes?" La teoría de la decisión es el estudio de cómo conviene tomar las decisiones frente a preguntas tales como “¿Subirán las tasas de interés? ¿Puedo confiar en tal persona?, si me opero de tal enfermedad ¿Cuál es el riesgo de morir?’”. Intentar explicar y describir como se realizan decisiones y cuales son las variables que determinan la conducta de elección de los seres humanos, en diversos contextos, es un asunto en parte psicológico, pero existe una teoría formal de la decisión que trata sobre las

Fecha recepción: 06/02/2007 • Fecha aceptación: 10/06/2007

Dirección de Postal:

Salguero $1692-8^{\circ}-\mathrm{A}$

Buenos Aires (1425)

Argentina

Teléfono: $54-11-4824-1753$

E-mail:ncortada@psi.uba.ar decisiones óptimas, es decir prescribe cuáles son las decisiones que conviene tomar en cada caso según los objetivos y la información de que se dispone.

La información que se tiene sobre los hechos a decidir es sumamente importante y en este sentido se pueden tomar decisiones bajo certeza, bajo completa ignorancia o con cierto riesgo. La toma de decisiones con toda certeza o con completa ignorancia no son problema, el caso más interesante es la toma de decisión con riesgo en el que se supone que el sujeto tiene alguna información y puede evaluar las probabilidades de los distintos estados de la naturaleza. Las elecciones con riesgo son en esencia "apuestas" cuyos resultados vienen determinados conjuntamente por la elección individual y algún procedimiento aleatorio específico. El que decide no puede saber qué estado del mundo se va a dar pero conoce las probabilidades de ocurrencia de los distintos estados. En algunos casos como en las apuestas con los juegos de dados se saben exactamente las probabilidades objetivas y se puede calcular la esperanza matemática de ganar o perder en un juego. En otros casos como en las inversiones en negocios, el jugar a la bolsa, etc., solo se conocen estimaciones subjetivas y aproximadas de las probabilidades, para poder calcular los beneficios. 
El estudio de los juicios humanos frente al riesgo y la incertidumbre se transformó desde 1970 cuando Kahneman y Tversky introdujeron su enfoque sobre heurística y sesgos y desafiaron los modelos que dominaban en la época y que eran estrictamente racionales. El enfoque de estos autores generó un torrente de investigaciones en psicología, a la cual se extendió y afectó el saber académico en economía, derecho, sociología, medicina y ciencias políticas. La importancia de estos problemas se puso en evidencia en el año 2002 cuando por primera vez en la historia de la psicología un psicólogo, el Dr. Daniel Kahneman, compartió el premio Nobel en Economía.

El núcleo de las ideas del programa de heurística y sesgos es que el juicio bajo incertidumbre se basa a menudo en una cantidad limitada de conceptos heurísticos simplificadores, más bien que en un procesamiento algorítmico más formal y extensivo, veamos con algún detalle esto. Los procedimientos utilizados en la resolución de un problema pueden ser por algoritmos o por heurísticos. Los algoritmos (viene de guarismo = cantidad y es una alteración por influjo del griego "arithmós") son estrategias que garantizan la solución; por ejemplo, un algoritmo son las reglas para realizar una división cualquiera de dos números, es decir lo que haríamos para dividir 240/30. Estas reglas nos darán un resultado indefectiblemente correcto. Los procedimientos heurísticos en cambio, (de "heurisko", yo hallo, descubro, en griego) (Corominas, 1973) son procedimientos que proveen ayuda en la solución de un problema, pero no de manera justificada. Son juicios intuitivos, que se basan en el conocimiento parcial, en la experiencia o en suposiciones que a veces son correctas y otras veces erradas, no existe una seguridad absoluta y lógica, sobre los mismos. Los investigadores citados trabajaron muchos años juntos, e identificaron tres heurísticos para propósitos generales, como veremos más adelante.

\section{ANTECEDENTES HISTÓRICOS}

El modelo clásico de elección racional que había sido aplicado sobre todo en Economía hasta los años 70, sostenía que la persona elige qué alternativa seguir, evaluando la probabilidad de cada resultado posible, determinando la utilidad que se deriva de cada una y combinando estas dos evaluaciones, la opción elegida será aquella que ofrece la combinación óptima de probabilidad y utilidad. Este cálculo de probabilidad y utilidad puede ser un juicio bastante difícil de lograr, pero la teoría de la elección racional supone que las personas lo hacen bien.

Sin embargo, existen muchas pruebas de que las evaluaciones de probabilidad y riesgos de las personas no suelen estar de acuerdo con las leyes de la probabilidad. Existen, además, algunas contribuciones psicológicas tempranas que han tenido importancia en la modificación de esta "teoría racional". Por ejemplo, el trabajo de Paul Meehl (1954) en el que comparó la predicción diagnóstica con métodos clínicos y estadísticos o actuariales y demostró que el método estadístico siempre obtenía mejores resultados. Por otro lado, Edwards (1963) introdujo los análisis de Bayes en la psicología y demostró que los juicios intuitivos de verosimilitud no se correspondían con el estándar formativo ideal. Finalmente Herbert Simon (1957) señaló que una total racionalidad suponía que el modelo de elección racional era un estándar poco realista para el juicio humano. Propuso un criterio más limitado para la realidad del accionar que llamó racionalidad limitada (bounded rationality) que reconocía en el proceso mental humano limitaciones inherentes; las personas eligen y razonan racionalmente, pero solamente dentro de las restricciones impuestas por su búsqueda limitada y sus capacidades de cálculo.

\section{EL PROGRAMA DE HEURÍSTICOS Y RIESGOS}

Inspirados en estos antecedentes Kahneman y Tversky (1973) desarrollaron su propia perspectiva de racionalidad limitada. Según estos autores los procesos de juicios intuitivos no solo eran más simples de los que exigían los modelos racionales sino que eran categóricamente de una clase diferente. Así describieron tres heurísticos con fines generales que son la accesibilidad, la representatividad y el anclaje o ajuste, que subyacen a muchos juicios intuitivos bajo incertidumbre. Aunque la intuición heurística se distingue de los procesos de razonamiento formativo por pautas de juicios sesgados, los heurísticos en sí mismos son procedimientos de estimación que de ningún modo son irracionales. Son respuestas intuitivas normales, no solo para los problemas de alta complejidad, sino para las más simples cuestiones de verosimilitud, frecuencia y predicción. Veamos ahora cuáles fueron algunos de los sesgos señalados por estos cognitivistas.

\section{EL SESGO DE LA LEY DE LOS PEQUEÑOS NÚMEROS}

En los trabajos de los primeros años estos investigadores (Tversky \& Kahneman, 1971) se dedicaron a demostrar que el pensamiento intuitivo de muchas personas sobre el concepto de azar era erróneo y ese error no solo se daba en personas con poca formación estadística sino que era compartido por muchos investigadores. Lo que llamaron la creencia en la ley de los pequeños números explica según ellos la conocida falacia de los jugadores. El jugador cree que la perfección de una moneda o de un dado le permite esperar que una desviación en un sentido (por ejemplo la aparición de cara varias veces seguidas) será compensada por una desviación en el otro (la aparición de cruz). Sin embargo, esto puede no ocurrir, porque cada tirada de un dado o de una moneda es una experiencia independiente. Sucede que algunos procesos naturales sí siguen estas leyes, por ejemplo un desvío en el equilibrio produce una fuerza que restablece el mismo. Pero las leyes del azar no operan de esta manera; los desvíos no se suprimen sino que a medida que una muestra aumenta el número de unidades, se van diluyendo.

La ley de los grandes números garantiza que las muestras grandes sean representativas de la población de la que se han extraído; pero no las muestras pequeñas. Sin embargo, la intuición de las personas también les hace pensar que las 
pequeñas cantidades se regirán por las leyes de los grandes números pero esto no es cierto. Kahneman y Tversky fueron también algunos de los primeros autores que señalaron la importancia de llevar a cabo los cálculos para analizar la potencia de una prueba estadística (Cohen 1969) para que la comunidad científica se protegiera de un rechazo apresurado de la hipótesis nula.

\section{LOS JUICIOS DE INCERTIDUMBRE}

Kahneman y Tversky (1973) fueron los primeros en señalar que al hacer pronóstico y juicios bajo incertidumbre las personas no parecen seguir los cálculos para el azar o la teoría estadística para la predicción. En vez de esto, se apoyan en una cantidad limitada de representaciones que algunas veces producen juicios razonables y otras conducen a errores sistemáticos grandes y a menudo no pueden interpretar las leyes de la regresión a la media. Estos autores consideran que los análisis sobre la incertidumbre (Kahneman y Tversky, 1982) en filosofía, estadística y teoría de la decisión, a veces consideran a la incertidumbre en términos de una sola dimensión de la probabilidad o grados de creencia; pero que una perspectiva psicológica revela que esta comprende una gran variedad de procesos y experiencias, ya que todos los días se toman decisiones. Generalmente el análisis de la decisión distingue entre elecciones con riesgo y sin riesgo. El ejemplo paradigmático es la decisión riesgosa de aceptar una apuesta que produce resultados momentáneos con una probabilidad específica. Por otro lado, una decisión sin riesgo es aceptar una transacción en la cual se cambia un bien o un servicio por dinero o trabajo.

Las distorsiones o anomalías que se registran en este sector del pensamiento humano (Thaler, 1992) pueden ser estudiados experimentalmente. Estos fenómenos han sido comparados con las ilusiones perceptivas como por ejemplo la de MüllerLyer o la del cubo de Necker (Piattelli, 1995) en tanto se trata en ambos casos de distorsiones en el ajuste del pensamiento al medio. Recordemos que la ilusión de Müller-Lyer consiste en cierta confianza de la percepción visual generada por dos líneas paralelas como las de la Figura 1.
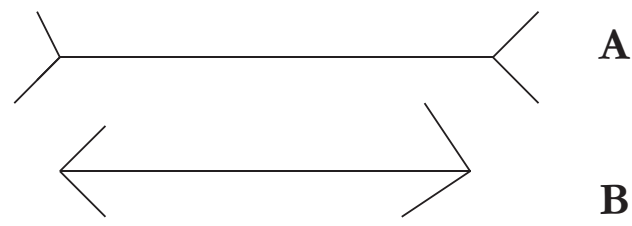

Figura 1. Ilusión de Müller-Lyer

Las líneas A y B parecen distintas a pesar de tener la misma longitud, porque la ilusión se genera por la forma en que se presentan las flechas de los extremos. Las distorsiones en el procesamiento humano no ocurren solo en el ámbito perceptivo, las decisiones de incertidumbre constituyen probablemente, uno de los sectores más estudiados desde esta perspectiva.

\section{LA ELECCIÓN RIESGOSA}

Es la que se hace sin el conocimiento adelantado de las consecuencias, como salir con paraguas o no. Dado que las consecuencias de tales acciones dependen de hechos inciertos, como el clima, este tipo de elección puede considerarse como la aceptación de una apuesta que puede tener distintos resultados con diferentes probabilidades. El enfoque de la elección riesgosa, iniciado por Bernouilli en 1738, deriva muchas de sus hipótesis de un análisis psicológico de las respuestas dadas bajo una cierta probabilidad de ganar dinero. Para ilustrar el análisis de Bernouilli, consideremos la elección ante la perspectiva de una probabilidad de 0,85 de ganar $\$ 1000$ (contra la probabilidad del 0,15 de no ganar nada) frente a la alternativa de recibir sin apostar $\$ 800$. Una gran mayoría de personas prefiere la seguridad, es decir recibir el dinero sin apostar, aunque la apuesta tiene una esperanza matemática más elevada es decir, haciendo el cálculo, (0,85 x 1000) + $(0,15 \times 0)=850$.

Esta preferencia por una ganancia segura es un caso de lo que los autores llaman (risk aversión) es decir rechazo al riesgo. La preferencia por el riesgo se llama búsqueda de riesgo (risk seeking). Bernouilli sugirió que las personas no tienen en cuenta las perspectivas de recibir el dinero, sino la esperanza del valor subjetivo de estos resultados. El valor subjetivo de una apuesta es también un promedio ponderado; pero ahora es el valor subjetivo de cada resultado el que se pondera por su probabilidad. Para explicar el rechazo al riesgo Bernouilli dijo que el valor subjetivo o utilidad es una función cóncava del dinero En tal función una diferencia de utilidad entre 200 y 100 es mayor que la diferencia de utilidad entre 1200 y 1100 . Por lo tanto, el valor subjetivo agregado a los $800 \$$ del ejemplo anterior, es más que el 80\% del valor de ganancia sobre 1000 . Esto hace que se dé un rechazo al riesgo en donde se podría ganar el 85\%, para aceptar una probabilidad de ganar el $80 \%$ de 1000 .

\section{LA TEORÍA DE LA EXPECTATIVA (PROSPECT THEORY)}

Los autores que estudiamos, Tversky y Khaneman (1983) señalan que las personas piensan en términos de ganancias, pérdidas y resultados neutrales. Así, proponen un análisis psicofísico de ganancias y pérdidas, y no en resultados totales, y este supuesto es lo que para considerar el tratamiento de la elección riesgosa dichos autores han llamado prospect theory 0 teoría de la expectativa. De este modo, al tener en cuenta que el valor subjetivo de una pérdida de 200 a 100, es mayor que el valor subjetivo de una pérdida de 1200 y 1100 , y haciendo una representación gráfica de ambos casos juntos, tenemos una curva en forma de S (con mayor pendiente para pérdidas que para ganancias.) (Véase la Figura 2). 


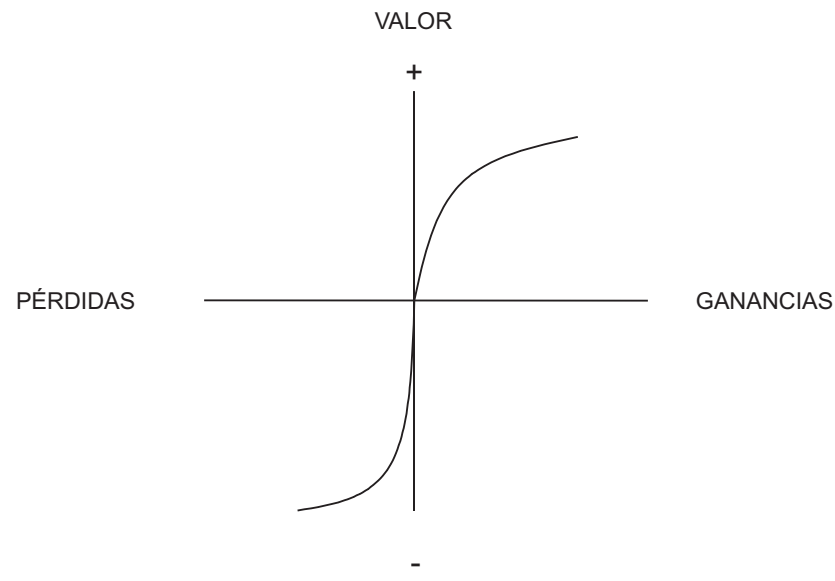

Fig. 2: Función Hipotética del Valor Subjetivo (Tomada de Kabneman \& Tversky, 2000)

Esta propiedad de generar mayor pendiente en la curva para pérdidas que para ganancias, se llama risk aversión o rechazo al riesgo, y hace que una pérdida de $\mathrm{X} \$$ produzca más rechazo que atracción una ganancia de los mismos X\$. El supuesto del rechazo al riesgo tiene gran importancia para la teoría económica. La concavidad de los valores de las ganancias supone rechazo al riesgo, mientras que la convexidad de los valores de pérdida supone búsqueda de riesgo. La mayoría de las personas prefieren la apuesta de perder 1000 (probabilidad de 0,85 de perder, y 0,15 de no perder) cuya esperanza matemática es $-0,85$, que una pérdida segura de -800 .

\section{USO COMÚN DEL HEURÍSTICO ACCESIBILIDAD}

Uno de los heurísticos más comunes es el de la accesibilidad. Esto significa que con frecuencia las personas valoramos la probabilidad o frecuencia de aparición de algún acontecimiento sobre la base de asociaciones que se tienen en mente. Por ejemplo para demostrar esto, Tversky y Khaneman utilizaban problemas como el siguiente:

"Se ha realizado un test de personalidad a 30 ingenieros y a 70 abogados, todos personas exitosas en sus respectivas carreras. Teniendo en cuenta esto considere la siguiente descripción, elegida al azar de las 100 disponibles y diga cuál es la probabilidad de que la siguiente descripción corresponda a un ingeniero:

Ricardo es un hombre de 35 años. Es casado sin hijos. Una persona de gran capacidad y motivación que promete tener éxito en su trabajo. Es muy bien visto por sus colegas"

La mayor parte de los examinados, psicólogos que habían seguido un curso de estadística, contestaron que la probabilidad de que Ricardo fuera ingeniero era del $50 \%$. No se daban cuenta de que si la muestra era de 100 y solo se habían examinado 30 ingenieros, la probabilidad real de ser ingeniero no podía ser mayor de 30/100.

\section{LOS PROBLEMAS DE DOMINANCIA E INVARIANCIA}

Otro problema estudiado por nuestros autores en relación a las elecciones racionales, se relacionan con dos principios que son conocidos desde el trabajo pionero de Von Neuman y Morgerstein (1948) y que se analizan sobre la teoría de la decisión. Estos don principios son el de la dominancia e invariancia.

La dominancia exige que si una expectativa $\mathrm{A}$ es por lo menos tan buena como la B en todos los aspectos y mejor que B al menos en un aspecto, A debe preferirse a B. La invariancia supone que el orden de preferencia entre dos expectativas no debe depender de cómo son descritas. Esto hace que dos versiones de un problema de elección que son equivalentes cuando se presentan juntas, deben demostrar la misma preferencia cuando se presentan por separado. Pero esto no suele ocurrir en los hechos. En los experimentos de Kahneman, debido al rechazo por el riesgo en un caso y a la preferencia por la búsqueda de riesgo en otro, los sujetos no respetaban el principio lógico de la invariancia. Uno de los problemas presentados por Kahneman (2000) es el siguiente:

"En un país X se cree que puede haber una epidemia de una enfermedad que podría matar a 600 personas. Se proponen varios programas sanitarios para combatir la epidemia. Las estimaciones de las consecuencias de cada programa son las siguientes:

\section{Primera Parte}

Programa A. Se podrán salvar 200 personas

Programa B. Existe una probabilidad de $1 / 3$ de que se salven las 600 personas y $2 / 3$ de que no se salve nadie.

\section{Segundaparte}

Otros investigadores presentaron los siguientes programas: Programa C. Si se adopta morirán 400 personas

Programa D. Existe una probabilidad de $1 / 3$ de que nadie muera y $2 / 3$ de probabilidad de que las 600 personas mueran.

En la primera parte del problema se habla de ganancias, medidas por el número de vidas salvadas y como era de esperar la mayoría de los sujetos $(72 \%)$ prefirió el programa A que representa rechazo al riesgo. En cambio en la segunda parte se habla de pérdidas de vida y en este caso las personas en su gran mayoría (78\%) eligen el programa D buscando apostar y aceptando la búsqueda de riesgo. Con esto se demuestra el fracaso del principio de la invarianzcia porque, como se puede ver observando los datos de los programas A y $\mathrm{C}$ son iguales, en uno se salvaran 200 de las 600 personas y en el otro morirán 400 .

\section{EL SESGO DE SOBRECONFIANZA}

El sesgo de sobreconfianza puede ser definido como un error sistemático de calibración subjetiva de éxito en la toma de decisiones bajo incertidumbre (Camerer \& Lovallo, 2000; 
Oskamp, 1965). Este error consiste en la sobreestimación del éxito de las propias decisiones. De manera más específica puede formularse como el exceso de confianza en las estimaciones subjetivas (E) en comparación con los resultados reales obtenidos u observados $(\mathrm{O})$ Esta relación puede apreciarse a continuación en la Ecuación 1.

$$
C=\sum_{i=1}^{n}\left(E_{i}-O_{i}\right)
$$

La confianza (C) o calibración subjetiva de éxito en las propias decisiones surge de la discrepancia entre lo estimado (E) y lo observado (O) para una serie (n) de tareas. Si lo estimado es mayor que lo observado, se genera el sesgo de sobreconfianza. Si lo estimado es menor que lo observado se produce el fenómeno contrario, esto es, el sesgo de subconfianza. La sobreconfianza se identifica con puntajes positivos en $\mathrm{C}$ y la subconfianza por puntajes negativos. En general todos los autores suelen hablar de un sesgo de sobreconfianza. Sin embargo, nosotros hicimos un experimento muy simple aplicando un test de conocimientos generales bastante difícil (Macbeth \& Cortada, 2005) Al comparar los puntajes obtenidos por los sujetos $(\mathrm{n}=79)$ con su estimación no hallamos diferencias significativas, es decir no hallamos sobreconfianza. Sin embargo cuando separamos el grupo de sujetos en dos subgrupos los que tenían resultados muy buenos y los que tenían resultados muy bajos encontramos que los sujetos de muy bajo rendimiento efectivamente sobreestimaban sus resultados (sobreconfianza) mientras que los sujetos de alto rendimiento, en cambio, subestimaban sus resultados. De todos modos esto son resultados provisorios pero seguimos trabajando en el tema, ampliando la cantidad de sujetos de las muestras y usando otros instrumentos de medición.

\section{LA FALACIA DE CONJUNCIÓN}

Las leyes de la probabilidad derivan de consideraciones extensivas. En estas se sabe que la probabilidad compuesta $\mathrm{P}(\mathrm{A}$ y $\mathrm{B})<\mathrm{P}(\mathrm{B})$ y $\mathrm{P}(\mathrm{A}$ y $\mathrm{B})<\mathrm{P}(\mathrm{A})$ Es decir que aunque $\mathrm{A}$ y $\mathrm{B}$ sean hechos independientes, una intersección no puede ser nunca más probable que uno de los constituyentes. En cambio los juicios intuitivos de probabilidad no son extensivos y muchas veces estas evaluaciones naturales para estimar o predecir un hecho que se llaman juicios heurísticos no son correctos. Los heurísticos pueden incluir similitud, representatividad, atribuciones de causalidad y también la falacia de conjunción que consiste en que alguna vez se llega a estimar que la probabilidad de un hecho B pueda ser menor que la probabilidad de A y B juntos. En uno de los problemas presentados se daba la descripción de un personaje "Linda" a un grupo de estudiantes, de la siguiente manera:
"Linda" es una chica de 31 años, soltera muy inteligente. Cuando era estudiante estaba muy preocupada por temas de discriminación y justicia social. Señale a continuación de las siguientes alternativas, 1 y 2 , cuál le parece más probable

1) Linda es ahora empleada de un banco

2) Linda es empleada de un banco y una activista femenina"

Más del 85\% de los encuestados señalaba la segunda alternativa como la más probable, (siendo así que la posesión de dos atributos juntos es siempre menos probable que la de uno solo). En este caso los examinados se equivocaban, usando la falacia de conjunción.

La incertidumbre es un aspecto inevitable de la condición humana. Muchas elecciones se basan sobre creencias, sobre la verosimilitud de los hechos de dudosa certidumbre como la culpa de un acusado, el resultado de una elección, el valor futuro del dólar, el resultado de una operación quirúrgica, la elección de una escuela para los hijos, etc., etc. El hombre no puede calcular, en muchos casos, formalmente las probabilidades y debe conformarse con su juicio intuitivo; pero este a menudo puede llevarle a errores y son precisamente estos errores los que es conveniente conocer para poder evitarlos. La familiaridad es también una decisión heurística automática. Se sabe que la familiaridad con un estímulo aumenta la respuesta afectiva, y las impresiones afectivas están siempre a mano y proporcionan una base más fácil para las decisiones que una evaluación cognitiva deliberada de cada opción. Esto no implica de ningún modo un desmerecimiento de nuestras capacidades para pensar. Recordemos que tampoco en la percepción se pueden soslayar algunas veces los sesgos como en las célebres figuras de las dos líneas paralelas de Müller-Lyer.

Las propuestas de Tversky y Kahneman han hecho reflexionar mucho a los psicólogos. En realidad, como suele ocurrir, también estos autores han recibido fuertes críticas. Por ejemplo Gigerenzer (1996) y Cosmides y Tolby (1996), y otros señalan que algunos problemas como el de Linda exigen opinar sobre la probabilidad de un suceso único y habría que saber cuál es la probabilidad de que Linda sea cajera de un banco, pero esto es incalculable y por eso los sujetos se equivocan tanto, pues el problema está en el enunciado en términos de frecuencia y de esto se seguirá que el modo de razonar de los que se equivocan no es de ningún modo ilógico.

No es posible en esta presentación analizar todos los problemas sobre elecciones riesgosas, decisiones sobre incertidumbre, etc., estudiados por esta rama del cognitivismo. Debemos señalar que estos estudios han representado y representan un gran estímulo para la realización de una serie de investigaciones sobre los temas más diversos que van desde el estudio de los juicios sociales (Kahneman \& Miller, 1986) a las elecciones que se hacen en el dominio de las emociones (Millar \& Schwards, 1999) lo cual indica la importancia de sus teorías y experiencias. 


\section{REFERENCIAS}

Bernouilli, D. (1954) Exposition of a new theory on the measurement of risk Econometrika 22, 23-36. (Publicación original 1738).

Camerer, C., \& Lovallo, D. (2000). Overconfidence and Excess Entry. En D. Kahneman \& A. Tversky (Eds.), Choices, Values and Frames (pp. 414-423). NewYork, USA: Cambridge University Press.

Cohen, J. (1969). Statistical analysis in the behavioral sciences. New Yoek, USA: Academic Press.

Corominas (1973). Breve diccionario etimológico de la lengua castellana. Madrid, España: Gredos.

Cortada, N., \& Macbeth, G. (2004). El reconocimiento público a la psicología cognitiva (A propósito del Premio Nobel otorgado en el 2002 a Daniel Kahneman). Irice, 18, 139164.

Cosmides, L. \& Tollby, J. (1956). Are humans good intuitive statisticians after all? Cognition, 58, 1-73.

Edwards, W., Lindman, H., \& Savage, L. J. (1963). Bayesian statistical inference for psychological research. Psychological Review, 70, 193-242.

Gigerenzer, J. (1996). On narrow norm and vague heuristics. A reply to Kahneman and Tversky (1996). Psychological Review, 103, 592-596.

Gigerenzar, G., Czerlinski, J., \& Martignon, L. (2002) How good are fast and frugal heuristics? En T. Gilovich, D. Griffin \& D. Kagneman (Eds.), Heuristics and Biases. New York, USA: Cambridge University Press.

Gilovich, T., Griffin, D., \& Kahneman, D. (Eds) (2003). Heuristics and Biases New York, USA: Cambridge University Press.

Kahneman, D., \& Tversky, A. (1973). The psychology of prediction. Psychological Review, 80(4), 237-251.
Kahneman, D., \& Tversky, A. (2000). Choices, Values and Frames. New York, USA: Russell Sage Foundation Cambridge University Press.

Kahneman, D., \& Miller, D. T. (1986). Norm theory. Comparing reality to its alternative Psychological Review, 93(6), 136-157.

Macbeth, G. \& Cortada, N. (2005). La estimación subjetiva de éxito en la toma de decisiones bajo incertidumbre. Actas de las Quintas Jornadas de Tecnología Aplicada a la Educación Matemática Universitaria de la Facultad de Ciencias Económicas de la UBA.

Meehl, P. E. (1954). Clinical versus statistical prediction. Minneapolis, USA: University Press.

Mellers, N., Schwartz, A., \& Ritor, I. (1999). Emotion Base Voice. Journal of Experimental Psychology, 128(3), 332-345.

Oskamp, S. (1965). Overconfidence in case-study judgments. The Journal of Consulting Psychology, 12, 269-275.

Piattelli, M. (1995). Los túneles de la mente ¿Qué se esconde tras nuestros errores? Barcelona, España: Crítica.

Simón, H. A. (1957). Models of man: Social and rational. New York: Wiley.

Thaler, R. H. (1992). The winner's Curse. Paradoxes and Anomalies of Economic Life. Princeton, USA: Princeton University Press.

Tversky, A., \& Kahneman, D. (1971). Belief in the law of small numbers. Psychological Bulletin, 76(2), 105-110.

Tversky, A., \& Kahneman, D. (1983). Extensional versus Intuitive reasoning. The Conjunction Fallacy in probability judgment. Psychological Review, 90(4), 293-315.

Von Neuman, J., \& Morgenstern, D. (1947). Theory of games and economic behavior. Princeton, USA: Princeton University Press. 